\section{Assessment of Genetic Diversity of Chinese Sand Pear Landraces (Pyrus pyrifolia Nakai) Using Simple Sequence Repeat Markers}

\author{
Zhengwang Jiang and Feiyan Tang \\ Wuhan Botanical Garden/Wuhan Institute of Botany, Chinese Academy of \\ Sciences, Wuhan, Hubei 430074, China
}

\begin{abstract}
Hongwen Huang ${ }^{1}$
South China Botanical Garden/South China Institute of Botany, Chinese Academy of Sciences, Guangzhou, Guangzhou, Guangdong 510650, China
\end{abstract}

\author{
Hongju Hu and Qiliang Chen \\ Institute of Fruit and Tea, Hubei Academy of Agricultural Sciences, Wuhan, \\ Hubei 430209, China
}

Additional index words. Pyrus pyrifolia, microsatellites, landrace, genetic diversity, sand pear germplasm

\begin{abstract}
The sand pear (Pyrus pyrifolia Nakai) is an important fruit crop in China. In this study, simple sequence repeats (SSRs) were used to estimate the level and pattern of genetic diversity among 233 sand pear landraces collected from 10 different geographic regions in China. The results demonstrated that the SSR technique is an effective tool for assessing genetic diversity and the geographic pattern of genetic variation among sand pear landraces of different origins. A total of 184 putative alleles was detected using 14 primer pairs with an average of $\mathbf{1 3 . 1}$ alleles per locus. The mean expected heterozygosity and observed heterozygosity across all loci were 0.705 and 0.671 , respectively. High genetic diversity was found in all populations except for that originated from Jiangxi $\left(A_{\mathrm{e}}=3.149 ; H_{\mathrm{e}}=0.655\right)$, whereas at the regional level, those from central China were less diverse than those from other regions. Analysis of molecular variance showed that most genetic differences resided among landraces within populations. Additionally, unweighted pair group with arithmetic average clustering and principal component analysis plotting based on Nei's genetic distance revealed distinct gene pools in agreement with geographic distribution.
\end{abstract}

The sand pear (Pyrus pyrifolia Nakai) is one of the most important fruit tree crops in China and is extensively cultivated in central and southwest China. The species occurs naturally in southern and western China, recognized as the center of origin of the genus Pyrus (Rubtsov, 1944). There are very many landraces (local cultivars) of $P$. pyrifolia owing to nearly 3000 years of cultivation and the complex climatic and geographical variation in China. Many landraces have unique traits. For example, 'Puguali', from Zhejiang Province, is a large-fruited cultivar with a mean weight of $553 \mathrm{~g}$ and a maximum weight of $950 \mathrm{~g}$. The skin is green when mature and covered with brown russet, which turns reddish brown when fruit are fully ripe. 'Cangxili', named after its place of origin, Cangxi county in Sichuan Province, is a traditional landrace

Received for publication 2 Oct. 2007. Accepted for publication 6 Jan. 2008.

This research was supported by the CAS project KSCX2-YW-N-061 and NSFC grant 30070082.

${ }^{1}$ To whom reprint requests should be addressed; e-mail huanghw@mail.scbg.ac.cn. age, $321.3 \mathrm{~g}$ ), smooth skin, and crisp and tender flesh, which is sweet and juicy and of high quality. The rich genetic resources in sand pear provide great potential for cultivar improvement and enhancement of the sustainability of the pear industry. However, many traditional local cultivars have been threatened with extinction by the changes that have occurred in the modern Chinese fruit industry over the past three decades. There has been large-scale cultivation of a few elite cultivars and top-grafting or replacement of old cultivars or landraces. This genetic loss could lead to serious erosion of the gene pool of the cultivated sand pear. To conserve and manage the diversity of sand pear landraces and cultivars, the Wuhan Sand Pear Germplasm Repository (WSPGR) was established in 1986 as the national repository for sand pears, and since then, an exhaustive collection of local cultivars and landraces of Chinese sand pear has been assembled.

Molecular techniques are useful tools for evaluating genetic diversity and for defining genetic relationships in fruit tree crops. In pear, chloroplast polymerase chain reactionrestriction fragment length polymorphisms with maximum fruit weight of $1850 \mathrm{~g}$ (aver-
(RFLPs) were used to examine relationships between east Asian species (Iketani et al., 1998). Dominant nuclear markers, random amplified polymorphic DNA (RAPD), amplified fragment length polymorphism (AFLP), and intersimple sequence repeat (ISSR) were used in an investigation of genetic relationships among species and for pear cultivar fingerprinting (Cao et al., 2007; Shen et al., 2006; Teng et al., 2001, 2002; Zhang et al., 2007), and nuclear and chloroplast DNA sequences have been used to identify pear cultivars (Kimura et al., 2003; Lee et al., 2004). Of the DNA marker systems currently available, simple sequence repeats (SSRs) have been considered one of the most useful for assessment of genetic diversity and cultivar fingerprinting at the intraspecific level because of their abundance, hyperpolymorphism, and codominant inheritance (Morgante and Olivieri, 1993; Tautz, 1989). However, the published work to date reported the use of a set of SSRs isolated from apple for verifying the transferability of SSRs between apple and pear (Yamamoto et al., 2001) and the cultivar identification by SSRs in a limited number of cultivars developed in Japan (Kimura et al., 2002). SSR markers have been proved as a robust tool for revealing genetic diversity in sand pear (Cao et al., 2007; Kimura et al., 2002, 2003), red-skin sand pear (Zhang et al., 2007), and some other pears in west China (Fan et al., 2007).

Current germplasm evaluation in China mostly focuses on morphological descriptions and documenting pomological traits. The exchange of plants between repositories or commercial orchards raises problems in that some individual sand pear landraces or cultivars may be known by several different names or the one name may be used for different landraces or cultivars. The information from current evaluation of sand pears was not sufficient. Furthermore, detailed morphological descriptions and comparisons of plants are time-consuming and fruitrelated traits cannot be observed until plants are mature to produce fruit. Genetic characterization of the gene pool of the cultivated sand pears in the WSPGR collection has not previously been attempted but is urgently needed for formulating management strategies for the WSPGR and for furnishing useful genetic information for future sand pear breeding efforts. This should provide a better understanding of the genetic diversity that exists in the gene pool of the cultivated sand pears and the diverse sources of useful genes in the germplasm repository. Therefore, the objectives of the present investigation were to determine the genetic diversity of the overall gene pool of sand pear landraces and assess the genetic variation among sand pear landrace groups in relation to their geographical distribution in China.

\section{Materials and Methods}

A total of 233 landraces originating from 10 provinces (designated as populations in this study) was obtained from the WSPGR 


\begin{tabular}{|c|c|c|c|c|}
\hline \multirow[b]{2}{*}{$\begin{array}{l}\text { Accession } \\
\text { number }\end{array}$} & \multirow[b]{2}{*}{ Landrace } & \multicolumn{3}{|c|}{ Original place of collection } \\
\hline & & District & $\begin{array}{c}\text { Province } \\
\text { (population) }\end{array}$ & Region \\
\hline 001 & Fu'andaxueli & Fu'an & Fujian & East China \\
\hline 002 & Qingpizhongli & Jian'ou & Fujian & East China \\
\hline 003 & Aijiali & Jianyang & Fujian & East China \\
\hline 004 & Bingzili & Jianyang & Fujian & East China \\
\hline 005 & Huangpizhongli & Jianyang & Fujian & East China \\
\hline 006 & Chipili & Jinjiang & Fujian & East China \\
\hline 007 & Eli & Jinjiang & Fujian & East China \\
\hline 008 & Pingguoli & Jinjiang & Fujian & East China \\
\hline 009 & Shuili & Jinjiang & Fujian & East China \\
\hline 010 & Bannannvli & Pingnan & Fujian & East China \\
\hline 011 & Mandingxueli & Pucheng & Fujian & East China \\
\hline 012 & Puchengxueli & Pucheng & Fujian & East China \\
\hline 013 & Panli & Shouning & Fujian & East China \\
\hline 014 & Liuyuehuangzongli & Shunchang & Fujian & East China \\
\hline 015 & Mali & Shunchang & Fujian & East China \\
\hline 016 & Baiyu & Jiujiang & Jiangxi & East China \\
\hline 017 & Hehua & Shangrao & Jiangxi & East China \\
\hline 018 & Huangpixiao & Shangrao & Jiangxi & East China \\
\hline 019 & Kuixingmake & Shangrao & Jiangxi & East China \\
\hline 020 & Xihuapingtouqing & Shangrao & Jiangxi & East China \\
\hline 021 & Jiangwanxipili & Wuyuan & Jiangxi & East China \\
\hline 022 & Bayuexue & Wuyuan & Jiangxi & East China \\
\hline 023 & Wuyuanbaili & Wuyuan & Jiangxi & East China \\
\hline 024 & Wuyuansuli & Wuyuan & Jiangxi & East China \\
\hline 025 & Wuyanxueli & Wuyuan & Jiangxi & East China \\
\hline 026 & Yousu & Wuyuan & Jiangxi & East China \\
\hline 027 & Danenli & Leqing & Zhejiang & East China \\
\hline 028 & Dahuangren & Leqing & Zhejiang & East China \\
\hline 029 & Huangqieli & Leqing & Zhejiang & East China \\
\hline 030 & Jiuzhongli & Leqing & Zhejiang & East China \\
\hline 031 & Puguali & Leqing & Zhejiang & East China \\
\hline 032 & Yandangxueli & Leqing & Zhejiang & East China \\
\hline 033 & Zhenxiangli & Lishui & Zhejiang & East China \\
\hline 034 & Hanghong & Unknown & Zhejiang & East China \\
\hline 035 & Huahong & Unknown & Zhejiang & East China \\
\hline 036 & Yuanli & Unknown & Zhejiang & East China \\
\hline 037 & Ruanxueli & Unknown & Zhejiang & East China \\
\hline 038 & Sanhua & Unknown & Zhejiang & East China \\
\hline 039 & Shanghaixueli & Unknown & Zhejiang & East China \\
\hline 040 & Nuodaoli & Yiwu & Zhejiang & East China \\
\hline 041 & Yiwulizi & Yiwu & Zhejiang & East China \\
\hline 042 & Zaosanhua & Yiwu & Zhejiang & East China \\
\hline 043 & Xihuaxueli & Yunhe & Zhejiang & East China \\
\hline 044 & Yunheshibianli & Yunhe & Zhejiang & East China \\
\hline 045 & Yunhexueli & Yunhe & Zhejiang & East China \\
\hline 046 & Fengkaihuizouli & Fengkai & Guangdong & South China \\
\hline 047 & Xinghuadayinli & Fengkai & Guangdong & South China \\
\hline 048 & Yeshenli & Fengkai & Guangdong & South China \\
\hline 049 & Hehuali & Fengkai & Guangdong & South China \\
\hline 050 & Gaoyaodanshuili & Gaoyao & Guangdong & South China \\
\hline 051 & Gaoyaohuangli & Gaoyao & Guangdong & South China \\
\hline 052 & Gaoyaoqingli & Gaoyao & Guangdong & South China \\
\hline 053 & Jianyeli & Gaoyao & Guangdong & South China \\
\hline 054 & Huiyangqingli & Huiyang & Guangdong & South China \\
\hline 055 & Huiyangsuanli & Huiyang & Guangdong & South China \\
\hline 056 & Xihuahongli & Huiyang & Guangdong & South China \\
\hline 057 & Xiangshuili & Huiyang & Guangdong & South China \\
\hline 058 & Qingpili & Lianping & Guangdong & South China \\
\hline 059 & Sianqingpili & Baise & Guangxi & South China \\
\hline 060 & Beiliuhuangli & Beiliu & Guangxi & South China \\
\hline 061 & Beiliumili & Beiliu & Guangxi & South China \\
\hline 062 & Beiliuqingli & Beiliu & Guangxi & South China \\
\hline 063 & Xiangjiaoli & Debao & Guangxi & South China \\
\hline 064 & Huangpichangbatangli & Gongcheng & Guangxi & South China \\
\hline 065 & Huangpisuanli & Gongcheng & Guangxi & South China \\
\hline 066 & Huangpixueli & Gongcheng & Guangxi & South China \\
\hline 067 & Qingpisuanli & Gongcheng & Guangxi & South China \\
\hline 068 & Xipitangli & Gongcheng & Guangxi & South China \\
\hline 069 & Cupitangli & Guanyang & Guangxi & South China \\
\hline 070 & Guanyanghongli & Guanyang & Guangxi & South China \\
\hline 071 & Guanyangqingshuili & Guanyang & Guangxi & South China \\
\hline 072 & Guanyangshuinanli & Guanyang & Guangxi & South China \\
\hline
\end{tabular}

(Continued on next page)
(Wuhan, China). Provinces were assigned to four regions (east, south, central, and southwest China). Details are given in Table 1 and Figure 1, and the numbers of landraces for each region and population are shown in Table 2.

Total genomic DNA was extracted from 1 to $2 \mathrm{~g}$ of fresh leaf tissue using the $2 \times \mathrm{CTAB}$ method of Doyle and Doyle (1987) with a slight modification $(3 \times \mathrm{CTAB})$. Extracted DNA was quantified in a BioPhotometer spectrophotometer (Eppendorf, Hamburg, Germany).

Sixteen SSR primer pairs developed from sand pear ( $P$. pyrifolia) (Yamamoto et al., $2002 \mathrm{~b}$ ) and two developed from apple (Gianfranceschi et al., 1998) were used for initial assay and of these,14 SSR primer pairs were chosen based on gel clarity and reliability of alleles (Table 3). Polymerase chain reactions were performed in a final volume of $10 \mu \mathrm{L}$ containing $20 \mathrm{~mm}\left(\mathrm{NH}_{4}\right)_{2} \mathrm{SO}_{4}, 1.5 \mathrm{~mm}$ $\mathrm{MgCl}_{2}, 0.1 \%$ Tween $20,75 \mathrm{~mm}$ Tris- $\mathrm{HCl}$ ( $\mathrm{pH} 8.8$ ), $0.2 \mathrm{~mm}$ dNTPs, $0.2 \mu \mathrm{M}$ of primer, 20 ng genomic DNA, and 0.5 unit Taq polymerase (Fermentas, Lithuania). The amplification was carried out in a PTC-200 thermal cycler (MJ Research, Watertown, MA) with the following protocol: $5 \mathrm{~min}$ at $95{ }^{\circ} \mathrm{C}$ followed by 35 cycles of $50 \mathrm{~s}$ at $94{ }^{\circ} \mathrm{C}, 50 \mathrm{~s}$ at $55^{\circ} \mathrm{C}$, and $50 \mathrm{~s}$ at $72{ }^{\circ} \mathrm{C}$ followed by a final 8 -min extension at $72{ }^{\circ} \mathrm{C}$. The amplification products with an aliquot 25 bp DNA ladder (Promega, Madison, WI) were electrophoresed in $6 \%$ denaturing polyacrylamide gel using a Sequi-Gen ${ }^{\circledR}$ GT/Power Pac 3000 Sequencer System (Bio-Rad, Hercules, CA) for $\approx 1.5 \mathrm{~h}$ at $55 \mathrm{~W}$ followed by a modified silver staining procedure (Sanguinetti et al., 1994). The bands of amplified DNA fragments were scored manually.

SSR markers were designated by the developer's primer code (referred to as locus) corresponding to the primer pair sequences followed by the product size (bp). Genetic diversity parameters were estimated: number of alleles per locus $(A)$, number of effective alleles per locus $\left(A_{\mathrm{e}}\right)$, observed heterozygosity $\left(H_{\mathrm{o}}\right)$, expected heterozygosity $\left(H_{\mathrm{e}}\right)$, and Shannon-Weaver's information index (I) using the program GenAlEx (Peakall and Smouse, 2001). The relative magnitude of genetic differentiation between geographic cultivar groups [Wright's $F$-statistic $\left(F_{\mathrm{ST}}\right)$ ] was calculated by different methods implemented in analysis of molecular variance (AMOVA) (Excoffier et al., 1992) and GenAlEx software. The power of discrimination $\left(P D=1-\sum p i^{2}\right.$, where $p_{i}$ is the frequency of the $i$-th genotype (Kloosterman et al., 1993), was also calculated for each locus. Nei et al.'s (1983) genetic distance $\left(D_{\mathrm{A}}\right)$ between populations was generated by the program POPULATION Version 1.2.28 (Olivier Langella; available at http://www.pge.cnrs-gif.fr/ bioinfo/populations). $D_{\mathrm{A}}$ has been considered the appropriate approach for phylogenetic analysis of closely related populations when SSR markers are used (Takezaki and Nei, 1996). The SAHN procedure of NTSYSpc2.0 software (Rohlf, 1997) was used to 


\begin{tabular}{|c|c|c|c|c|}
\hline \multirow[b]{2}{*}{$\begin{array}{l}\text { Accession } \\
\text { number }\end{array}$} & \multirow[b]{2}{*}{ Landrace } & \multicolumn{3}{|c|}{ Original place of collection } \\
\hline & & District & $\begin{array}{c}\text { Province } \\
\text { (population) }\end{array}$ & Region \\
\hline 073 & Guanyangtangli & Guanyang & Guangxi & South China \\
\hline 074 & Guanyangxueli & Guanyang & Guangxi & South China \\
\hline 075 & Guanyangzaoheli & Guanyang & Guangxi & South China \\
\hline 076 & Huangpieli & Guanyang & Guangxi & South China \\
\hline 077 & Quanzhouli & Guilin & Guangxi & South China \\
\hline 078 & Yanshanhuangpixiao & Guilin & Guangxi & South China \\
\hline 079 & Yanshanliuyuexue & Guilin & Guangxi & South China \\
\hline 080 & Yanshanqingpixueli & Guilin & Guangxi & South China \\
\hline 081 & Hengxianjinpaoli & Hengxian & Guangxi & South China \\
\hline 082 & Hengxianlingshanli & Hengxian & Guangxi & South China \\
\hline 083 & Hengxianmili & Hengxian & Guangxi & South China \\
\hline 084 & Nanningdashali & Hengxian & Guangxi & South China \\
\hline 085 & Jingxiqingpili & Jingxi & Guangxi & South China \\
\hline 086 & Jingxixueli & Jingxi & Guangxi & South China \\
\hline 087 & Shanggangli & Leye & Guangxi & South China \\
\hline 088 & Lipuhuangpili & Lipu & Guangxi & South China \\
\hline 089 & Lipuxueli & Lipu & Guangxi & South China \\
\hline 090 & Liuchengfengshanli & Liucheng & Guangxi & South China \\
\hline 091 & Liuchengxueli & Liucheng & Guangxi & South China \\
\hline 092 & Sanmenjianghuangli & Liucheng & Guangxi & South China \\
\hline 093 & Guihuali & Longsheng & Guangxi & South China \\
\hline 094 & Huangcupili & Longsheng & Guangxi & South China \\
\hline 095 & Huangxipili & Longsheng & Guangxi & South China \\
\hline 096 & Napoqingpili & Tianyang & Guangxi & South China \\
\hline 097 & Bingtangli & Unknown & Guangxi & South China \\
\hline 098 & Beiliuhuali & Wuming & Guangxi & South China \\
\hline 099 & Cangwudashali & Wuzhou & Guangxi & South China \\
\hline 100 & Badongjingli & Badong & Hubei & Central China \\
\hline 101 & Jianshichengtuoli & Jianshi & Hubei & Central China \\
\hline 102 & Jianshichixianfeng & Jianshi & Hubei & Central China \\
\hline 103 & Jianshizaoguli & Jianshi & Hubei & Central China \\
\hline 104 & Lichuanxiangshui & Lichuan & Hubei & Central China \\
\hline 105 & Suizhouzaoshuli & Suizhou & Hubei & Central China \\
\hline 106 & Huishuijingai & Unknown & Hubei & Central China \\
\hline 107 & Houzuili & Unknown & Hubei & Central China \\
\hline 108 & Huangpixiang & Unknown & Hubei & Central China \\
\hline 109 & Make & Unknown & Hubei & Central China \\
\hline 110 & Zaomili & Wuhan & Hubei & Central China \\
\hline 111 & Wanxianfeng & Xianfeng & Hubei & Central China \\
\hline 112 & Xianfengbaijie & Xianfeng & Hubei & Central China \\
\hline 113 & Xianfengchengtuoli & Xianfeng & Hubei & Central China \\
\hline 114 & Xianfenghongjuli & Xianfeng & Hubei & Central China \\
\hline 115 & Xianfengxuepingli & Xianfeng & Hubei & Central China \\
\hline 116 & Xianfengyangdongli & Xianfeng & Hubei & Central China \\
\hline 117 & Xxueping & Xianfeng & Hubei & Central China \\
\hline 118 & Xuanenchengtuoli & Xuan'en & Hubei & Central China \\
\hline 119 & Xuanenxueli(2) & Xuan'en & Hubei & Central China \\
\hline 120 & Xuanenyangdongli & Xuan'en & Hubei & Central China \\
\hline 121 & Jinbangtou & Yuan'an & Hubei & Central China \\
\hline 122 & Longtuanli & Yuan'an & Hubei & Central China \\
\hline 123 & Shilixiang & Yuan'an & Hubei & Central China \\
\hline 124 & Suanbiantou & Yuan'an & Hubei & Central China \\
\hline 125 & Wangshuibai & Yuan'an & Hubei & Central China \\
\hline 126 & Huaihuaxiangshui & Anjiang & Hunan & Central China \\
\hline 127 & Baojingyangdong & Baojing & Hunan & Central China \\
\hline 128 & Daguoqing & Jinxian & Hunan & Central China \\
\hline 129 & Tanghuqing & Jinxian & Hunan & Central China \\
\hline 130 & Yadianqing & Jinxian & Hunan & Central China \\
\hline 131 & Gengtouqing & Linwu & Hunan & Central China \\
\hline 132 & Qingli & Linwu & Hunan & Central China \\
\hline 133 & Shexiangli & Linwu & Hunan & Central China \\
\hline 134 & Xiangheli & Linwu & Hunan & Central China \\
\hline 135 & Xianghuali & Linwu & Hunan & Central China \\
\hline 136 & Zamali & Linwu & Hunan & Central China \\
\hline 137 & Longhuijuli & Longhui & Hunan & Central China \\
\hline 138 & Duanbazao & Yizhang & Hunan & Central China \\
\hline 139 & Qingpizao & Yizhang & Hunan & Central China \\
\hline 140 & Tianxiaoli & Yizhang & Hunan & Central China \\
\hline 141 & Meitanjingai & Meitan & Guizhou & Southwest China \\
\hline 142 & Meitanmugua & Meitan & Guizhou & Southwest China \\
\hline 143 & Fanli & Weining & Guizhou & Southwest China \\
\hline 144 & Weiningbaipili & Weining & Guizhou & Southwest China \\
\hline
\end{tabular}

construct the unweighted pair group using arithmetic average (UPGMA) dendrogram as described by Sneath and Sokal (1973). A principal coordinate analysis (Gower, 1966) was also conducted using the same software package.

\section{Results}

Simple sequence repeat polymorphism and fingerprinting sand pear landraces. Of the 16 primer pairs prescreened, 14 resolved clear SSR banding patterns, whereas those of NH012a were not adequately clear for allele scoring and CH01E12 failed to amplify in several samples. These two primer pairs were therefore discarded. The 14 loci generated a total of 184 alleles among 233 sand pear landraces, varying from six to 21 alleles per locus with an average of 13.1 alleles per locus (Table 3 ). The mean value of gene diversity $\left(H_{\mathrm{e}}\right)$ was 0.705 , ranging from 0.527 for NH005b to 0.825 for NH014a. The observed heterozygosity $\left(H_{\mathrm{o}}\right)$ ranged from 0.451 for NH013a to 0.844 for $\mathrm{NH004a}$ with a mean value 0.671 . The fixation index $(F)$, which estimates the degree of allelic fixation by comparison of $H_{\mathrm{e}}$ and $H_{\mathrm{o}}$, was 0.048 , suggesting predominantly outcrossing in sand pears. The mean Wright's $F_{\mathrm{ST}}$ was 0.129 , ranging from 0.097 to 0.156 (Table $3)$. The mean estimate of power of discrimination $\left(\mathrm{P}_{\mathrm{D}}\right)$ was relatively high with mean value of 0.91 and ranged from 0.77 for $\mathrm{NH} 005 \mathrm{~b}$ to 0.97 for NH014a. These results indicated that the 14 SSR loci used were efficient in distinguishing the 233 sand pear landraces.

A total of 20 unique alleles (alleles present in only one or two landraces) was found in eight populations, ranging from one allele in two landraces from both Jiangxi and Yunnan Provinces to five alleles in five different landraces from Guizhou Province (Table 4). At the geographical region level, southern and central China each has three unique alleles, whereas five and nine were found in eastern China and southwestern China, respectively. In addition, two unique alleles were detected in 'Xianfengbaijie', which came from Hubei Province in central China.

Genetic diversity within and among populations. At the population level, the mean number of alleles per locus $(A)$ ranged from 5.643 in Jiangxi Province to 8.500 in Guangxi and Guizhou, and the mean effective number of alleles per locus $\left(A_{\mathrm{e}}\right)$ was also lowest in Jiangxi (3.149) and highest in Guangxi (5.071) (Table 2). The estimates of expected heterozygosity $\left(H_{\mathrm{e}}\right)$ and ShannonWeaver's information index $(I)$ showed gene diversity to be highest in Guangxi $\left(H_{\mathrm{e}}=0.773, I=1.765\right)$ and lowest in Jiangxi $\left(H_{\mathrm{e}}=0.655, I=1.322\right)$ (Table 4$)$. At the regional level, landraces in central China were found to be genetically less diverse than those in other regions, whereas the eastern, southern, and southwestern China regions had similar levels of genetic diversity (Table 2). 
The estimate of Wright's $F_{\mathrm{ST}}$ across 10 Original place of collection

\begin{tabular}{|c|c|c|c|c|}
\hline & & & & \\
\hline $\begin{array}{l}\text { Accession } \\
\text { number }\end{array}$ & Landrace & District & $\begin{array}{c}\text { Province } \\
\text { (population) }\end{array}$ & Region \\
\hline 145 & Weiningbaiwali & Weining & Guizhou & Southwest China \\
\hline 146 & Weiningchengxiangli & Weining & Guizhou & Southwest China \\
\hline 147 & Weinigcili & Weining & Guizhou & Southwest China \\
\hline 148 & Weiningdahuangli & Weining & Guizhou & Southwest China \\
\hline 149 & Weininghuahong & Weining & Guizhou & Southwest China \\
\hline 150 & Weininghuapili & Weining & Guizhou & Southwest China \\
\hline 151 & Weininghuazhali & Weining & Guizhou & Southwest China \\
\hline 152 & Weininghuangshuili & Weining & Guizhou & Southwest China \\
\hline 153 & Weininghuangsuanli & Weining & Guizhou & Southwest China \\
\hline 154 & Weiningjingaili & Weining & Guizhou & Southwest China \\
\hline 155 & Weiningmali & Weining & Guizhou & Southwest China \\
\hline 156 & Weiningmianhuali & Weining & Guizhou & Southwest China \\
\hline 157 & Weiningmopanli & Weining & Guizhou & Southwest China \\
\hline 158 & Weiningmuguali & Weining & Guizhou & Southwest China \\
\hline 159 & Weiningpidaili & Weining & Guizhou & Southwest China \\
\hline 160 & Weiningqingli & Weining & Guizhou & Southwest China \\
\hline 161 & Weiningqingpimopan & Weining & Guizhou & Southwest China \\
\hline 162 & Weiningsuanqingli & Weining & Guizhou & Southwest China \\
\hline 163 & Weiningtiansuanli & Weining & Guizhou & Southwest China \\
\hline 164 & Weiningxiangmianli & Weining & Guizhou & Southwest China \\
\hline 165 & Weiningxiaohuangli & Weining & Guizhou & Southwest China \\
\hline 166 & Weiningzaibaili & Weining & Guizhou & Southwest China \\
\hline 167 & Weiningzaoli & Weining & Guizhou & Southwest China \\
\hline 168 & Weiningzishengli & Weining & Guizhou & Southwest China \\
\hline 169 & Xiaobaizhaotongli & Weining & Guizhou & Southwest China \\
\hline 170 & Yingxueli & Weining & Guizhou & Southwest China \\
\hline 171 & Xingyihaizi & Xingyi & Guizhou & Southwest China \\
\hline 172 & Zunyixueli & Zunyi & Guizhou & Southwest China \\
\hline 173 & Cangxixueli & Cangxi & Sichuan & Southwest China \\
\hline 174 & Damali & Cangxi & Sichuan & Southwest China \\
\hline 175 & Dahuali & Jianyang & Sichuan & Southwest China \\
\hline 176 & Jiangyangdabaili & Jianyang & Sichuan & Southwest China \\
\hline 177 & Chenjiadamali & Jinchuan & Sichuan & Southwest China \\
\hline 178 & Chengxiangdamali & Jinchuan & Sichuan & Southwest China \\
\hline 179 & Hongxiangli & Jinchuan & Sichuan & Southwest China \\
\hline 180 & Hanyuanbanjinli & Hanyuan & Sichuan & Southwest China \\
\hline 181 & Hanyuanshisheng(1) & Hanyuan & Sichuan & Southwest China \\
\hline 182 & Hanyuanzhaobaoli & Hanyuan & Sichuan & Southwest China \\
\hline 183 & Jinhuali & Hanyuan & Sichuan & Southwest China \\
\hline 184 & Ludingshishengli(2) & Hanyuan & Sichuan & Southwest China \\
\hline 185 & Shangengzifengli & Hanyuan & Sichuan & Southwest China \\
\hline 186 & Huilihuoba & Huili & Sichuan & Southwest China \\
\hline 187 & Huilishibingli & Huili & Sichuan & Southwest China \\
\hline 188 & Huilixianhuangli & Huili & Sichuan & Southwest China \\
\hline 189 & Huilizaobai & Huili & Sichuan & Southwest China \\
\hline 190 & Huilichangbali & Huili & Sichuan & Southwest China \\
\hline 191 & Huilixueli & Huili & Sichuan & Southwest China \\
\hline 192 & Ludingqingpifengli & Luding & Sichuan & Southwest China \\
\hline 193 & 1237 & Tongliang & Sichuan & Southwest China \\
\hline 194 & Baihuali & Tongliang & Sichuan & Southwest China \\
\hline 195 & Jindiaozi & Tongliang & Sichuan & Southwest China \\
\hline 196 & Jinzhuguoli & Tongliang & Sichuan & Southwest China \\
\hline 197 & Ruifu & Tongliang & Sichuan & Southwest China \\
\hline 198 & Zhuzuiba & Tongliang & Sichuan & Southwest China \\
\hline 199 & Baozhuli & Chenggong & Yunnan & Southwest China \\
\hline 200 & Dabaozhu & Chuxiong & Yunnan & Southwest China \\
\hline 201 & Huobali & Chuxiong & Yunnan & Southwest China \\
\hline 202 & Kunmingmali & Chuxiong & Yunnan & Southwest China \\
\hline 203 & Shuaili & Chuxiong & Yunnan & Southwest China \\
\hline 204 & Dalihuoba & Dali & Yunnan & Southwest China \\
\hline 205 & Dalisuanli & Dali & Yunnan & Southwest China \\
\hline 206 & Dalixueli & Dali & Yunnan & Southwest China \\
\hline 207 & Fuyuanhuangli & Fuyuan & Yunnan & Southwest China \\
\hline 208 & Xibaqingshuili & Kunming & Yunnan & Southwest China \\
\hline 209 & Haidongli & Lijiang & Yunnan & Southwest China \\
\hline 210 & Lijiangdazhonggu & Lijiang & Yunnan & Southwest China \\
\hline 211 & Lijiangdazhonggu & Lijiang & Yunnan & Southwest China \\
\hline 212 & Lijianghuangbali & Lijiang & Yunnan & Southwest China \\
\hline 213 & Lijianghuangsuanli & Lijiang & Yunnan & Southwest China \\
\hline 214 & Lijiangmazhanli(2) & Lijiang & Yunnan & Southwest China \\
\hline 215 & Lijiangmaniaoli & Lijiang & Yunnan & Southwest China \\
\hline 216 & Lijiangmianli & Lijiang & Yunnan & Southwest China \\
\hline
\end{tabular}

(Continued on next page) populations of sand pear landraces was 0.129 , suggesting that $\approx 87 \%$ of the total genetic variance resided within populations. Furthermore, AMOVA revealed that $88.60 \%$ of the total molecular variation was attributed to differentiation among individuals within populations, and only a small amount of variation was partitioned among regions $(3.85 \%)$ and among populations $(7.55 \%)$, yet there was a significant level of differentiation among regions and populations $(P<$ 0.01) (data now shown).

Genetic relatedness of sand pear landraces from different provinces and regions. $\mathrm{Nei}$ et al.'s (1983) genetic distance $\left(D_{\mathrm{A}}\right)$ for all possible pairwise population comparisons ranged from 0.450 between Guangdong and Guangxi to 0.792 between Guangdong and Jiangxi (Table 5). The UPGMA based on $D_{\mathrm{A}}$ values revealed three main clusters, which show clear genetic relationships reflecting their geographic distribution (Fig. 2). Each cluster was further subdivided into two subclusters; Cluster I was made up of three populations from east China, including Jiangxi, Zhejiang, and Fujian Provinces. Three populations from southwest China were grouped into Cluster II, whereas Cluster III has two subclusters consisting of two populations (Guangdong, Guangxi) in south and southwest China and two populations (Hubei, Hunan) in central China.

The relationships revealed by principal components analysis (PCA) were similar to those revealed by UPGMA. The first three components of the PCA explained more than $55 \%$ of the variation found in the $D_{\mathrm{A}}$ genetic distance matrix. The bidimensional scatterplot strongly differentiated geographically all of the populations except that from Jiangxi (Fig. 3). The first two components, which accounted for $27.62 \%$ and $14.39 \%$ of total variation, respectively, divided the 10 populations into three major groups and a single population, Jiangxi.

\section{Discussion}

Effective conservation of plant germplasm requires a thorough understanding of the existing genetic diversity in the species being studied, its genetic structure and geographic distribution and whether it is to be conserved ex situ or in situ (Allard, 1988; Hamrick and Godt, 1997). Molecular markers, for example, AFLP, RAPD, RFLP, ISSR, and SSR, have been used to determine relationships between a limited number of sand pear cultivars (Cao et al., 2007; Kimura et al., 2002; Shen et al., 2006; Teng et al., 2001, 2002; Zhang et al., 2007) but a genetic analysis of the extant gene pool made up from the large number of traditional landraces in China has not previously been attempted. The present study provides most of the information now available on the extent of genetic diversity within sand pear landraces, information that is required for germplasm repository management in China. As was also 
Table 1. (Continued) Chinese landraces of sand pear evaluated.

\begin{tabular}{lllll}
\hline & & \multicolumn{2}{c}{ Original place of collection } \\
\cline { 3 - 4 } $\begin{array}{l}\text { Accession } \\
\text { number }\end{array}$ & \multicolumn{1}{c}{ Landrace } & District & $\begin{array}{c}\text { Province } \\
\text { (population) }\end{array}$ & Region \\
\hline 217 & Lijiangpopulu & Lijiang & Yunnan & Southwest China \\
218 & Lijiangtapili & Lijiang & Yunnan & Southwest China \\
219 & Lijiangtianzhonggu & Lijiang & Yunnan & Southwest China \\
220 & Lijiangxiaohuangli & Lijiang & Yunnan & Southwest China \\
221 & Lijiangzhimali & Lijiang & Yunnan & Southwest China \\
222 & Suomeili & Lijiang & Yunnan & Southwest China \\
223 & Sangpili & Lijiang & Yunnan & Southwest China \\
224 & Zhonggushiyueli & Lijiang & Yunnan & Southwest China \\
225 & Miduhuangpili & Midu & Yunnan & Southwest China \\
226 & Midushenwali & Midu & Yunnan & Southwest China \\
227 & Miduxiaomianli & Midu & Yunnan & Southwest China \\
228 & Miduyuli & Midu & Yunnan & Southwest China \\
229 & Hongyunyihao & Unknown & Yunnan & Southwest China \\
230 & Hongyunerhao & Unknown & Yunnan & Southwest China \\
231 & Lushali & Unknown & Yunnan & Southwest China \\
232 & Qiubaili & Unknown & Yunnan & Southwest China \\
233 & Wenshanhongli & Unknown & Yunnan & Southwest China \\
\hline
\end{tabular}

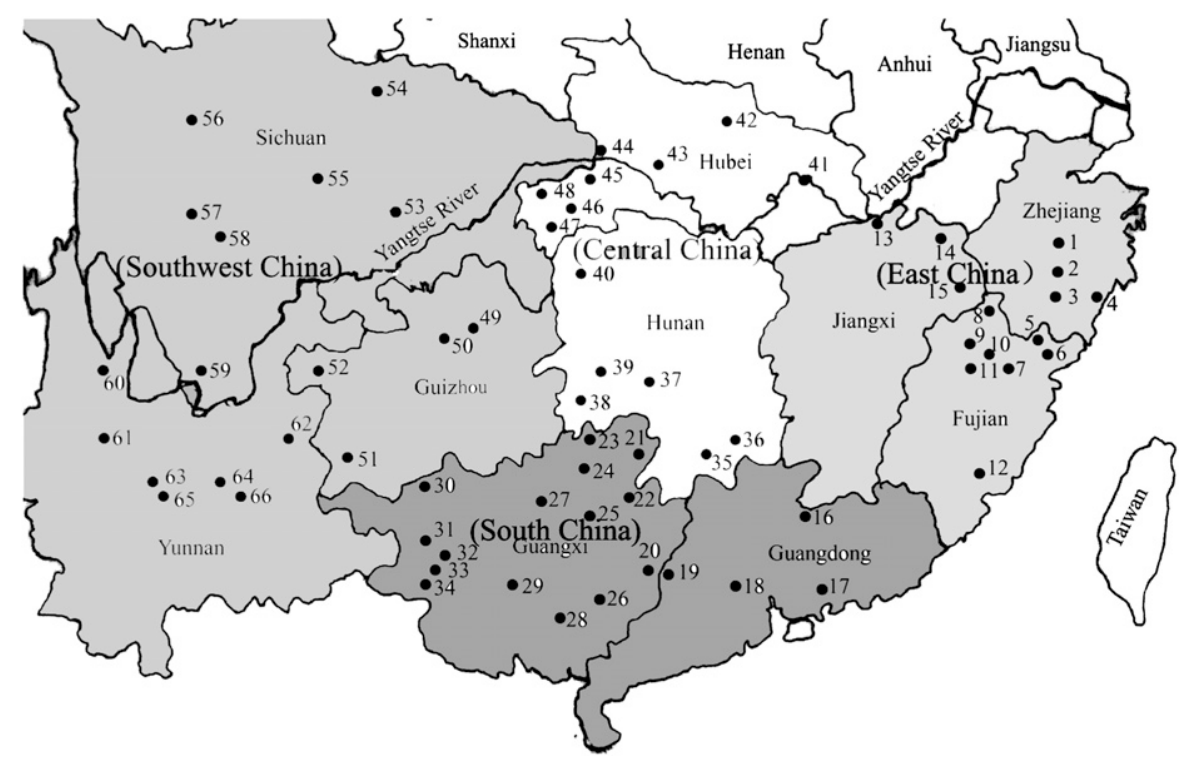

Fig. 1. Sixty-six geographic origins of 233 sand pear landraces evaluated in this study. 1) Yiwu; 2) Lishui 3) Yunhe; 4) Leqing; 5) Shouning; 6) Fuan; 7) Pingnan; 8) Pucheng; 9) Jianyang; 10) Jianou; 11) Shunchang; 12) Jinjiang; 13) Jiujiang; 14) Wuyuan; 15); Shangrao; 16) Lianping; 17) Huiyang; 18) Gaoyao; 19) Fengkai; 20) Wuzhou; 21) Guanyang; 22) Gongcheng; 23) Longsheng; 24) Guilin; 25) Lipu; 26) Beiliu; 27) Liucheng; 28) Hengxian; 29) Wuming; 30) Leye; 31) Baise; 32) Tianyang; 33) Debao; 34) Jingxi; 35) Linwu; 36) Yizhang; 37) Longhui; 38) Jingxian; 39) Anjiang; 40) Baojing; 41) Wuhan; 42) Suizhou; 43) Yuanan; 44) Badong; 45) Jianshi; 46) Xuanen; 47) Xianfeng; 48) Lichuan; 49) Meitan; 50) Zunyi; 51) Xingyi; 52) Weining; 53) Tongliang; 54) Cangxi; 55) Jianyan; 56) Jinchuan; 57) Luding; 58) Hanyuan; 59) Huili; 60) Lijing; 61) Dali; 62) Fuyuan; 63) Midu; 64) Kunming; 65) Chuxiong; 66) Chenggong.

found by Kimura et al. (2002), SSRs are particularly useful for assessing levels of genetic variation in cultivated sand pears. All except two of the primer pairs tested allowed reliable scoring of alleles across 233 landraces. The 14 primer pairs used all appear to be hypervariable and generated a total of 184 different alleles, which made it possible to discriminate unequivocally all the sand pear genotypes studied. The average estimates of genetic diversity for each locus $\left(A=13.1, H_{\mathrm{o}}=0.671, \mathrm{PD}=0.91\right)$ found in this study were slightly different from those $\left(A=14.8, H_{\mathrm{o}}=0.63, \mathrm{PD}=0.91\right)$ derived by Kimura et al. (2002) in their work on Pyrus species. The higher than expected heterozy- gosity $\left(H_{\mathrm{o}}\right)$ observed by us in sand pear landraces indicates that they are genetically more diverse than the 60 pear accessions from six Pyrus species studied by Kimura et al. (2002). However, the slightly lower value of $A$ could be the result of different sets of SSR primer pairs being used in the two studies and/or to unique alleles not present in P. pyrifolia.

The extent and distribution of genetic diversity within a plant species depends on its evolution and breeding systems, ecological and geographical factors, and human activities. Although sand pears were generally believed to lack morphological variation (Yamamoto et al., 2002a, 2002b), the high
SSR diversity observed in all populations appears to be related to the evolutionary history of the species. First, sand pears were and still are widely distributed throughout south-central China, including at least 13 provinces. Such a large number of sand pear landraces accumulated during the long history of selection and cultivation should have generated tremendous genetic variation in adaptation to different environments. This high genetic diversity would be maintained despite the intense but narrowly focused selection under the complex ecogeographic factors such as latitude, altitude, temperature, and rainfall. Second, the self-incompatibility mating system of $P$. pyrifolia could effectively prevent genetic homogenization within each population as evidenced by the highly discriminatory power of the SSR procedure (Table 3). The rich genetic diversity in sand pears provides an opportunity to broaden the genetic base of modern pear cultivars Although the Guangxi, Guizhou, Sichuan, and Yunnan populations were slightly more diverse genetically than the other populations, when all estimates of genetic diversity were considered, the difference was not significant except for the Jiangxi population being the least diverse (Table 2). The provincial populations with the highest mean number (8.500) of alleles per locus were those in Guizhou and Guangxi. The regional population with the most alleles per locus (11.428) was from southwestern China; this regional population also showed the highest values for $A e$ and $I$ (Table 2), suggesting that the Guizhou population should perhaps be a priority for conservation.

It is likely that the genetic diversity of the sand pear gene pool may have been underestimated because that the landraces used in this analysis were only some representatives from different parts of the geographic distribution (Table 1). For example, the 11 landraces in Jiangxi were collected from only three districts with six of them collected from Wuyuan and four and one from Shangrao and Jiujiang, respectively; $87.5 \%$ of the landraces (28 of 32 accessions) in Guizhou were derived from a single county, Weining, an area with a long history of pear cultivation (Fig. 1). Therefore, we can reasonably conclude that there are extensive sand pear resources yet to be collected and assessed. Further work should be focused on those districts that have not so far been studied considering the significant genetic differentiation among and between regions. A total of 20 unique alleles was found in 23 landraces belonging to eight different populations. These local alleles could be associated with certain adaptive characteristics, which would be of interest to plant breeders for cultivar improvement and should be given priority for conservation.

At the regional level, southwestern China contains the most diverse sand pear germplasm with slightly lower genetic diversity in central China (Table 2). Our results provide further support for the hypothesis that $P$. pyryfolia originated from southwest China, 
Table 2. Estimates of genetic diversity of sand pear in different provinces and regions.

\begin{tabular}{lcrccc}
\hline Population & $N^{z}$ & \multicolumn{1}{c}{$A^{\mathrm{y}} \pm \mathrm{SE}$} & $A_{\mathrm{e}}^{\mathrm{x}} \pm \mathrm{SE}$ & $H_{\mathrm{e}}^{\mathrm{w}} \pm \mathrm{SE}$ & $I^{v} \pm \mathrm{SE}^{\mathrm{u}}$ \\
\hline East China & 45 & $9.857 \pm 0.694$ & $5.080 \pm 0.404$ & $0.780 \pm 0.027$ & $1.824 \pm 0.080$ \\
Fujian & 15 & $7.500 \pm 0.511$ & $4.764 \pm 0.377$ & $0.773 \pm 0.018$ & $1.716 \pm 0.072$ \\
Jiangxi & 11 & $5.643 \pm 0.038$ & $3.149 \pm 0.258$ & $0.655 \pm 0.027$ & $1.322 \pm 0.066$ \\
Zhejiang & 19 & $7.429 \pm 0.500$ & $4.587 \pm 0.377$ & $0.747 \pm 0.018$ & $1.641 \pm 0.072$ \\
South China & 54 & $8.857 \pm 0.653$ & $5.249 \pm 0.425$ & $0.784 \pm 0.036$ & $1.794 \pm 0.096$ \\
Guangdong & 13 & $6.071 \pm 0.530$ & $3.917 \pm 0.380$ & $0.719 \pm 0.021$ & $1.482 \pm 0.085$ \\
Guangxi & 41 & $8.500 \pm 0.532$ & $5.071 \pm 0.541$ & $0.773 \pm 0.024$ & $1.765 \pm 0.090$ \\
Central China & 41 & $8.785 \pm 0.800$ & $4.115 \pm 0.437$ & $0.731 \pm 0.021$ & $1.617 \pm 0.086$ \\
Hubei & 26 & $7.857 \pm 0.762$ & $3.956 \pm 0.511$ & $0.714 \pm 0.023$ & $1.552 \pm 0.088$ \\
Hunan & 15 & $6.429 \pm 0.571$ & $3.832 \pm 0.369$ & $0.706 \pm 0.028$ & $1.477 \pm 0.093$ \\
Southwest China & 93 & $11.428 \pm 0.850$ & $5.400 \pm 0.507$ & $0.780 \pm 0.033$ & $1.863 \pm 0.099$ \\
Guizhou & 32 & $8.500 \pm 0.769$ & $4.953 \pm 0.541$ & $0.758 \pm 0.035$ & $1.711 \pm 0.111$ \\
Sichuan & 26 & $8.286 \pm 0.588$ & $4.628 \pm 0.548$ & $0.742 \pm 0.031$ & $1.675 \pm 0.097$ \\
Yunnan & 35 & $8.357 \pm 0.427$ & $4.380 \pm 0.360$ & $0.740 \pm 0.033$ & $1.651 \pm 0.082$ \\
\hline
\end{tabular}

${ }^{\mathrm{z}}$ Number of landraces.

${ }^{y}$ Mean number of alleles per locus (for each region, all the alleles were considered).

${ }^{x}$ Effective number of alleles per locus.

"Nei's expected heterozygosity.

'Shannon-Weaver's information index.

${ }^{\mathrm{u}} \mathrm{SD}$.

Table 3. Simple sequence repeat (SSR) primers and estimates of SSR polymorphism parameters based on 233 Chinese sand pear landraces.

\begin{tabular}{lcccccc}
\hline Locus code & Size range $(\mathrm{bp})$ & $A$ & $H_{\mathrm{e}}$ & $H_{\mathrm{o}}$ & $\mathrm{V}$ & PD \\
\hline NH001c & $118-160$ & 9 & 0.661 & 0.500 & 0.154 & 0.88 \\
NH002b & $148-200$ & 13 & 0.718 & 0.733 & 0.152 & 0.92 \\
NH004a & $78-130$ & 17 & 0.748 & 0.844 & 0.124 & 0.86 \\
NH005b & $304-350$ & 9 & 0.527 & 0.554 & 0.136 & 0.77 \\
NH007b & $120-152$ & 15 & 0.679 & 0.738 & 0.130 & 0.88 \\
NH008b & $188-215$ & 10 & 0.747 & 0.669 & 0.120 & 0.93 \\
NH009b & $138-170$ & 16 & 0.694 & 0.518 & 0.136 & 0.92 \\
NH011b & $151-196$ & 18 & 0.736 & 0.695 & 0.133 & 0.94 \\
NH013a & $190-225$ & 12 & 0.695 & 0.451 & 0.106 & 0.90 \\
NH014a & $60-130$ & 21 & 0.825 & 0.802 & 0.097 & 0.97 \\
NH015b & $100-136$ & 13 & 0.715 & 0.740 & 0.116 & 0.94 \\
NH017a & $88-120$ & 13 & 0.717 & 0.731 & 0.156 & 0.94 \\
CH01F02 & $163-184$ & 12 & 0.743 & 0.672 & 0.106 & 0.95 \\
CH01H10 & $97-123$ & 6 & 0.666 & 0.747 & 0.136 & 0.90 \\
Mean & & 13.1 & 0.705 & 0.671 & 0.129 & 0.91 \\
\hline
\end{tabular}

${ }^{\mathrm{z}}$ Developed from apple (Gianfranceschi et al., 1998).

Table 4. Unique simple sequence repeat alleles in eight pear landrace populations.

\begin{tabular}{llcl}
\hline Population & Locus & Allele (bp) & \multicolumn{1}{c}{ Landrace (no.) $^{\mathrm{z}}$} \\
\hline Fujian & NH004a & 83 & Eli (007) \\
& NH014a & 110 & Panli (013) \\
Jiangxi & NH009b & 164 & Jiangwanxipili (021), Yousu (026) \\
Zhejiang & NH004a & 130 & Jiuzhongli (030) \\
& NH011b & 172 & Zhenxiangli (033) \\
Guangxi & NH008b & 206 & Quanzhouli (077) \\
& NH009b & 148 & Cangwudashali (099) \\
& CH01F02 & 176 & Yanshanhuangpixiao (078) \\
Guizhou & NH004a & 98 & Weiningzaoli (167) \\
& NH007b & 146 & Weiningxiaohuangli (165) \\
& NH009b & 138 & Yingxueli (170) \\
& CH01F02 & 172 & Weiningbaipili (144) \\
Hubei & CH01F02 & 184 & Weiningqingpimopan (161) \\
& NH002b & 148 & Make (109), Xianfengbaijie (112) \\
& NH004a & 123 & Xianfengbaijie (112) \\
Sichuan & NH017a & 120 & Lichuanxiangshui (104), Wangshuibai (125) \\
& NH002b & 160 & Huilixianghuangli (188) \\
& NH007b & 136 & Hanyuanzhaobaoli (182) \\
Yunnan & NH015b & 132 & Shangengzifengli (185) \\
\hline
\end{tabular}

${ }^{\mathrm{z}}$ Numbers in parentheses are the accession numbers listed in Table 1.

including Guizhou, Sichuan, and Yunnan Provinces (Rubtsov, 1944). It is interesting to note that the sand pear populations in eastern China (Fujian, Jiangxi, and Zhejiang) are more diverse than those in central China (Hubei and Hunan). This might be the result of ancient gene flow through cultivar exchanges by local pear growers in different regions of China. Historically, pear production in eastern China was closely associated with Japan and Korea, where sand pears are also distributed, and probably provided addi- tional opportunities for genetic exchanges with foreign cultivars developed in Japan. RAPD analysis of Pyrus species and cultivars revealed close genetic relationships between cultivars from Fujian, Zhejiang, and Japan, indicating ancient gene flow among those regions (Teng et al., 2002).

Analysis of molecular variance showed that most genetic diversity of landraces was within populations with smaller, although still significant, amounts of genetic variation between regions and populations. The estimate of Wright's $F_{\mathrm{ST}}(0.129)$ was also higher for sand pears than for wild populations of plant species with similar life histories $\left(F_{\mathrm{ST}}=\right.$ 0.084, Hamrick et al., 1992). This fact reflects the outstanding characteristic of landraces; they are the result of selection through climatic and edaphic adaptation and human activities (Harlan, 1975a, 1975b). Indeed, local crop races are the consequences of long periods of interaction between the environment and genetic systems (Brush, 1995). During the long history of cultivation, farmers have made direct selection for desirable genotypes under the specific agricultural regimes resulting in accumulated genetic differentiation among populations.

The UPGMA dendrograms show that the 10 populations formed region-specific clusters (Fig. 2). Three populations in east China grouped into a distinct subcluster, suggesting profound genetic divergence from other populations, which might be the result of the $P$. pyrifolia distribution. In addition, landraces in this region maybe have an ancient gene introgression with genotypes from Japan and/ or Korea, which further increased differentiation with landraces of inland China. Similar region-specific clusters were also evident in the PCA plots, except that the Jiangxi population cannot be grouped with the Fujian and Zhejiang populations (Fig. 3). Furthermore, the Fujian population was, surprisingly, more similar to the Guizhou and Yunnan populations than to its two bordering populations in Jiangxi and Guangdong as shown by the genetic distance matrix (Table 5) and PCA plots (Fig. 3). One possible explanation is that the landraces collected from those populations were from the same latitude and similar climate conditions may force landraces into similar ecotypes. Populations from Hubei and Hunan, Guangdong and Guangxi, and Guizhou, Yunnan, and Sichuan generated similar clusters in the UPGMA dendrograms and PCA plots, which reflected their geographic relationships. The three distinct clusters of the 233 landraces suggest that east China, south China, and southwest China have different germplasm pools and should each be conserved.

In summary, the present study has demonstrated that SSR markers provide an effective tool for assessing genetic diversity and relationships within sand pear germplasm. Further work should focus on combining molecular data and pomological traits for delineating a core collection of sand pear and developing effective conservation strategies and breeding programs. 
Table 5. Genetic distance ( $\mathrm{D}_{\mathrm{A}}$, Nei et al., 1983) matrix of 10 populations of Pyrus pyrifolia in China.

\begin{tabular}{lcccccccccc}
\hline Population & Fujian & Jiangx. & Zhej. & Guangd. & Guangx. & Hube. & Hun. & Guiz. & Sichu. & Yunn. \\
\hline Fujian & 0.000 & & & & & & & & & \\
Jiangxi & 0.659 & 0.000 & & & & & & & & \\
Zhejiang & 0.548 & 0.472 & 0.000 & & & & & & & \\
Guangdong & 0.603 & 0.792 & 0.555 & 0.000 & & & & & & \\
Guangxi & 0.610 & 0.722 & 0.529 & 0.450 & 0.000 & & & & & \\
Hubei & 0.641 & 0.767 & 0.577 & 0.598 & 0.475 & 0.000 & & & & \\
Hunan & 0.645 & 0.779 & 0.611 & 0.582 & 0.574 & 0.520 & 0.000 & & & \\
Guizhou & 0.562 & 0.744 & 0.505 & 0.575 & 0.544 & 0.522 & 0.619 & 0.000 & & \\
Sichuan & 0.648 & 0.733 & 0.564 & 0.613 & 0.584 & 0.613 & 0.615 & 0.595 & 0.000 & \\
Yunnan & 0.535 & 0.744 & 0.527 & 0.541 & 0.519 & 0.549 & 0.567 & 0.506 & 0.465 & 0.000 \\
\hline
\end{tabular}

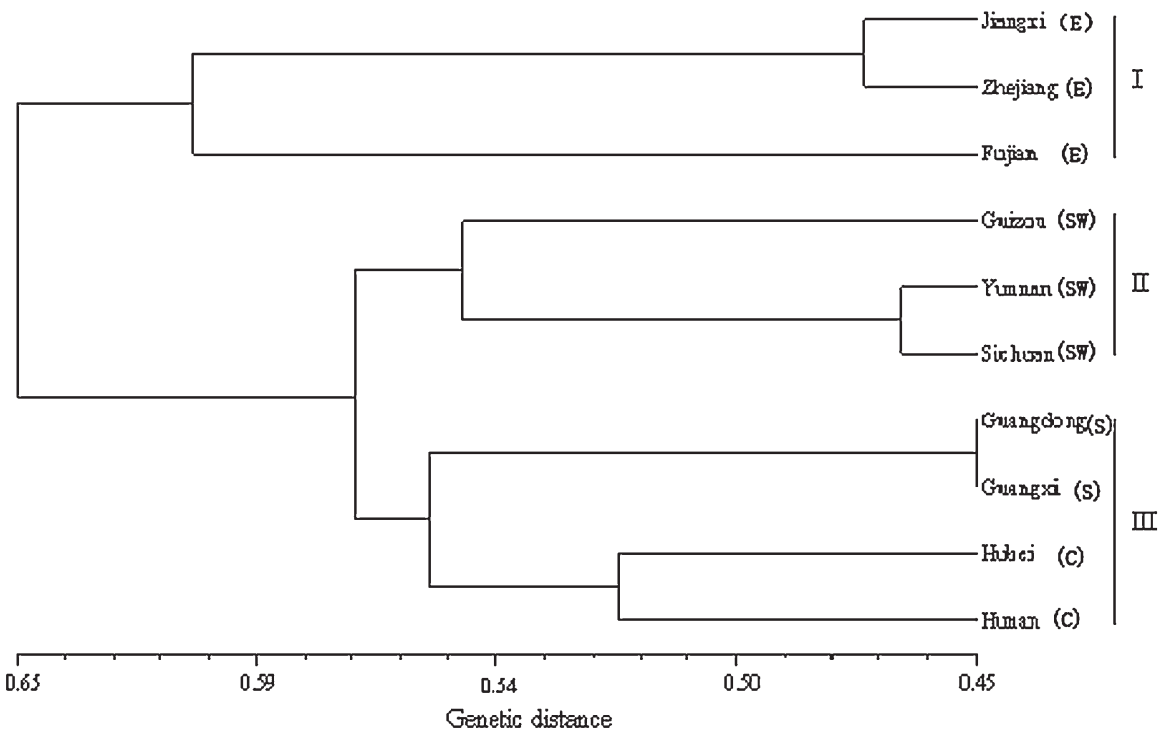

Fig. 2. Unweighted pair group with arithmetic average dendrograms of 10 populations of sand pear based on Nei et al.'s (1983) genetic distance $\left(D_{\mathrm{A}}\right)$.

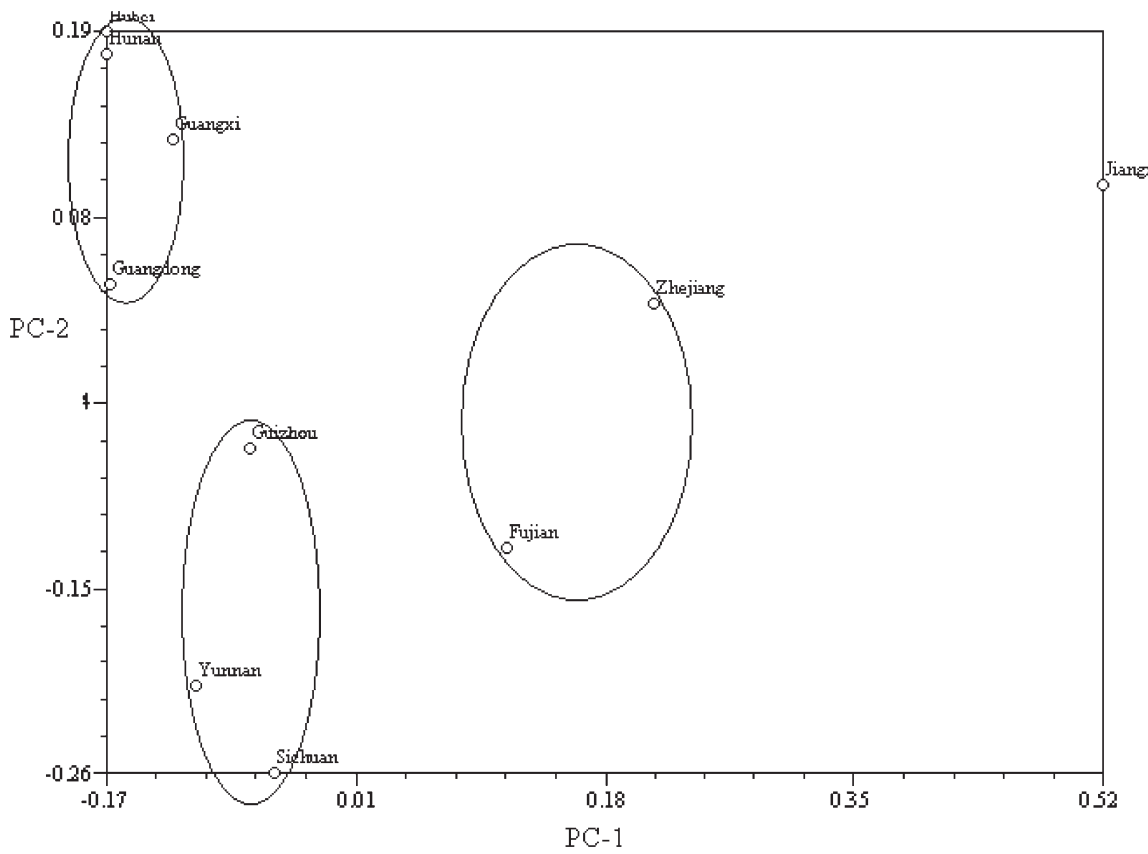

Fig. 3. Plot of first and second principal components of principal components analysis on 10 populations of sand pear, together accounting for $42.01 \%$ of the total variation.

\section{Literature Cited}

Allard, R.W. 1988. Genetic changes associated with the evolution of adaptedness in cultivated plants and their wild progenitors. J. Hered. 79:225-238.
Brush, S.B. 1995. In situ conservation of landraces in centres of crop diversity. Crop Sci. 35:346354.

Cao, Y.F., F.Z. Liu, Y. Gao, L.J. Jiang, K. Wang, Z.Y. Ma, and K.C. Zhang. 2007. SSR analysis of genetic diversity of pear cultivars. Acta Hort. Sinica 34:305-310.

Doyle, J.J. and J.L. Doyle. 1987. A rapid DNA isolation procedure for small quantities of fresh leaf tissue. Phytochemical Bulletin 19:11-15.

Excoffier, L., P.E. Smouse, and J.M. Quattro. 1992. Analysis of molecular variance inferred from metric distances among DNA haplotypes: Application to human mitochondrial DNA restriction sites. Genetics 131:479-491.

Fan, T.W., D.Y. Cai, H.X. Li, F.L. Wang, C.Z. Zhao, and Y.W. Teng. 2007. Simple sequence repeat (SSR) analysis for assessment of genetic variation and relationships in pear germplasm native to the middle area of Gansu province. J. Fruit Science 24:268-275.

Gianfranceschi, L., N. Seglias, and R. Tarchini. 1998. Simple sequence repeats for the genetic analysis of apple. Theor. Appl. Genet. 96:1069-1076.

Gower, J.C. 1966. Some distance properties of latent root and vector methods used in multivariate analysis. Biometrika 53:325-338.

Hamrick, J.L. and M.J.W. Godt. 1997. Allozyme diversity in cultivated crops. Crop Sci. 37:26-30.

Hamrick, J.L., M.J.W. Godt, and S.L. ShermanBroyles. 1992. Factors influencing levels of genetic diversity in woody plant species. New For. 6:95-124.

Harlan, J.R. 1975a. Geographic patterns of variability in some cultivated plants. J. Hered. 66:184-191.

Harlan, J.R. 1975b. Our vanishing genetic resources. Science 188:618-621.

Iketani, H., T. Manabe, N. Matsuta, T. Akihama, and T. Hayashi. 1998. Incongruence between RFLPs of chloroplast DNA and morphological classification in east Asian pear (Pyrus spp.). Gene. Resour. Crop Ev. 45:533-539.

Kimura, T., H. Iketani, K. Kotobuki, N. Matsutan, Y. Ban, T. Hayashi, and T. Yamamoto. 2003. Genetic characterization of pear varieties revealed by chloroplast DNA sequences. J. Hort. Sci. Biotechnol. 78:241-247.

Kimura, T., Z.S. Yong, M. Shoda, K. Kotobuki, N. Matsuta, T. Hayashi, Y. Ban, and T. Yamamoto. 2002. Identification of Asian pear varieties by SSR analysis. Breed. Sci. 52:115-121.

Kloosterman, A.D., B. Budowle, and P. Daselaar. 1993. PCR amplification and detection of the human D1S80 VNTR locus amplification conditions, population genetics and application in forensic analysis. Intl. J. Legal Med. 105:257-264.

Lee, G.P., C.H. Lee, and C.S. Kim. 2004. Molecular markers derived from RAPD, SCAR, and the conserved 18S rDNA sequences for classification and identification in Pyrus pyrifolia and $P$. communis. Theor. Appl. Genet. 108:1487-1491.

Morgante, M. and A. Olivieri. 1993. PCR-amplified microsatellites as markers in plant genetics. Plant J. 3:175-182.

Nei, M., F. Tajima, and Y. Tateno. 1983. Accuracy of estimated phylogenetic trees from molecular data. J. Mol. Evol. 19:153-170.

Peakall, R. and P.E. Smouse. 2001. GenAlEx V5.1: Genetic analysis in Excel. Population genetic software for teaching and research. Australian National University, Canberra, Australia. <http:// www.anu.edu.au/BoZo/GenAlEx/>.

Rohlf, F.J. 1997. NTSYS-pc: Numerical taxonomy and multivariate analysis system. Version 2.0. Exeter Software, Setauket, NY

Rubtsov, G.A. 1944. Geographical distribution of the genus Pyrus and trends and factors in its evolution. Am. Nat. 78:358-366.

Sanguinetti, C.J., N.E. Dias, and A.J.G. Simpson. 1994. Rapid silver staining and recovery of PCR products separated on polyacrylamide gels. Biotechniques 17:915-919. 
Shen, Y.Y., Y.W. Teng, and K. Tanabe. 2006. RAPD analysis for genetic assessment of some cultivars of Pyrus pyrifolia derived from China and Japan. Acta Hort. Sinica. 33:621624.

Sneath, P.H.A. and R.R. Sokal. 1973. Numerical taxonomy. Freeman, San Francisco, CA.

Takezaki, N. and M. Nei. 1996. Genetic distances and reconstruction of phylogenetic trees from microsatellite DNA. Genetics 144:389 399.

Tautz, D. 1989. Hypervariability of simple sequences as a general source for polymorphic DNA markers. Nucleic Acids Res. 17:64636471.
Teng, Y.W., K. Tanabe, F. Tamura, and A. Itai. 2001. Genetic relationships of pear cultivars in Xinjiang, China as measured by RAPD markers. J. Hort. Sci. Biotechnol. 76:771-779.

Teng, Y.W., K. Tanabe, F. Tamura, and A. Itai. 2002. Genetic relationships of Pyrus species and cultivars native to East Asia revealed by randomly amplified polymorphic DNA markers. J. Amer. Soc. Hort. Sci. 127: 262-270.

Yamamoto, T., T. Kimura, Y. Sawamura, K. Kotobuki, Y. Ban, T. Hayashi, and N. Matsuta. 2001. SSRs isolated from apple can identify polymorphism and genetic diversity in pear. Theor. Appl. Genet. 102:865-870.
Yamamoto, T., T. Kimura, Y. Sawamura, T. Manabe, K. Kotobuki, T. Hayashi, Y. Ban, and N. Matsuta. 2002a. Simple sequence repeats for genetic analysis in pear. Euphytica 124:129-137.

Yamamoto, T., T. Kimura, M. Shoda, Y. Ban, T. Hayashi, and N. Matsuta. 2002b. Development of microsatellite markers in the Japanese pear (Pyrus pyrifolia Nakai). Mol. Ecol. Notes 2: 14-16.

Zhang, D., Q. Shu, Y.W. Teng, M.H. Qiu, L. Bao, and H.J. Hu. 2007. Simple sequence repeat analysis on genetic assessment of Chinese red skinned sand pear cultivars. Acta Hort. Sinica 34:47-52. 